\title{
Improved Reset Control Design for a PZT Positioning Stage
}

\author{
Jinchuan Zheng, Yuqian Guo, Minyue Fu, Youyi Wang, and Lihua Xie
}

\begin{abstract}
Reset control was studied for enhanced performance that can not be obtained by linear controllers. The conventional reset control is simple for implementation by resetting some of its controller states to zero when its input is zero. However, we find that in some cases the performance of conventional reset control is still limited such as only partial reduction of the overshoot. Thus, this paper presents the stability analysis and design of an improved reset control system, where the reset times are prespecified and the controller states are reset to certain non-zero values, which are calculated online in terms of the system states for optimal performance. Experimental results on a PZT microactuator positioning stage show that the improved reset control can completely remove the overshoot and thus achieve shorter settling time than the conventional reset control. Moreover, robustness tests against various step levels, disturbance and sensor noise are presented.
\end{abstract}

\section{INTRODUCTION}

Reset control was firstly proposed by Clegg [1] to overcome limitations of linear control. This reset controller, termed as Clegg integrator, consists of an integrator and a reset law which resets the amount of integration to zero when its input crosses zero. From the basic idea of reset control, one can see that reset control is capable of reducing windup caused by integration. Moreover, a Clegg integrator has a similar magnitude-frequency slope as a pure integrator, but with $51.9^{\circ}$ less phase lag. This favorable property helps to enlarge phase margin of a system. Krishman and Horowitz have developed a quantitative control design procedure in [2] for Clegg integrator and have generalized the concept of reset control to higher order systems [3]. Related works on this have been also summarized in [4], [5].

A lot of works show the advantages of reset control over linear control. For instance, an example is presented in [6] showing that reset control can achieve some control specifications, which cannot be achieved by any ordinary linear control. Moreover, it is experimentally demonstrated in [7] that reset control can achieve better senor noises suppression while without degrading disturbance rejection nor losing of gain or phase margin. These advantages make reset control effective for performance improvement in other applications [8]-[10].

Typically, there are two steps in reset control design [11]: linear compensator design and reset element design. Linear

J. Zheng and M. Fu are with the School of Electrical Engineering and Computer Science, the University of Newcastle, Callaghan, NSW 2308, Australia Jinchuan. Zheng@newcastle. edu . au ; Minyue.Fu@newcastle.edu.au

Y. Guo, Y. Wang and L. Xie are with the School of Electrical and Electronic Engineering, Nanyang Technological University, Singapore 639798 YQGuoantu. edu.sg; EYYWangantu.edu.sg; ELHXie@ntu.edu.sg compensator is firstly designed to meet all performance specifications except for the overshoot constraint. Then reset element is designed to meet the overshoot specification. However, the reset controller can improve the closed-loop performance only when the reset law interacts well with the base linear system. In other words, if the reset controller is not appropriately designed, it may have little contribution to the performance improvement, or even cause performance degradation. In reset control system design, there are three basic problems: stability analysis, base linear system design and reset law design.

For stability analysis, there are lots of literatures addressing this issue such as [12]-[15]. Most of these existing results require stability of the base linear system. In fact, stability of a reset control system is related to both the base linear system and the reset actions. Either factor may destroy the stability of the whole system. Note that reset control systems are also known as impulsive systems. Many stability results which do not rely on the stability of the base linear systems have been obtained in [16].

For reset control design, more efforts are put on the design of base linear system in existing literatures. The reset law adopted is generally the conventional one, i.e., resetting the controller states to zero when the controller input crosses zero. The base linear system is then designed to interact well with the reset law. We refer to this kind of reset control as conventional reset control in this paper. The conventional reset control has been demonstrated to be able to achieve better performance than a pure linear controller. However, we find that in some cases the performance of conventional reset control is still limited, for example, the overshoot is only partially reduced based on the linear control system [7], [8]. Actually, reset control can be more generalized by relaxing the design of the reset times and the reset values to push the performance improvement further.

The purpose of this paper is to propose an improved reset control system, where the reset times can be prespecified and the controller states are reset to certain non-zero values, which are calculated online in terms of the system states for optimal performance. In Section II, we firstly formulate the improved reset control system in a state space form. Then, the reset control system is reformulated as a new system, which is referred to as induced difference system. We show that under some mild conditions the stability of a reset control system is equivalent to the stability of its induced difference system. Based on this, we obtain some new results about stability of reset control systems, which do not rely on the stability of base linear system. Lastly, we propose a reset law design approach, which aims at 


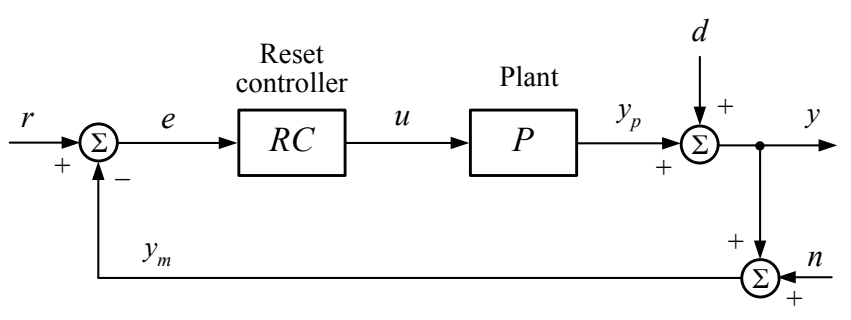

Fig. 1. Block diagram of a reset control system.

minimizing a performance index related to the tracking error. Section III demonstrates our proposed reset control design on a piezoelectric (PZT) microactuator positioning stage. Experimental results showed its effectiveness in overshoot removal, disturbance and sensor noise suppression. Conclusions and future works are given in Section IV.

\section{IMPROVED RESET CONTROL DESIGN}

\section{A. Formulation of Reset Control Systems}

A typical reset control system is depicted in Fig. 1, where $r$ is the reference input, $e$ is the feedback error, $u$ is the reset controller output, $d$ is the output disturbance, $n$ is the sensor noise, and $y_{p}, y$ and $y_{m}$ denote the plant output, controlled output and measurement output, respectively. The linear plant $P$ is described by

$$
\Sigma_{P}:\left\{\begin{array}{l}
\dot{x}_{p}=A_{p} x_{p}+B_{p} u \\
y_{p}=C_{p} x_{p}
\end{array}\right.
$$

where $x_{p} \in \mathbb{R}^{n_{p}}, u \in \mathbb{R}$, and $y_{p} \in \mathbb{R}$. The reset controller (RC) is described by impulsive differential equation (IDE) as follows:

$$
\Sigma_{R C}: \begin{cases}\dot{x}_{r}=A_{r} x_{r}+B_{r} e, & t \neq t_{k} \\ x_{r}\left(t_{k}^{+}\right)=E_{k} x_{p}+F_{k} x_{r}+G_{k} r, & t=t_{k} \\ u=C_{r} x_{r}+D_{r} e & \end{cases}
$$

where $x_{r} \in \mathbb{R}^{n_{r}}$ is the reset controller state, $e \in \mathbb{R}$, and $t_{k}^{+}$is the reset time. $A_{r}, B_{r}, E_{k}, F_{k}, G_{k}, C_{r}$, and $D_{r}$ are appropriate dimensional constant matrices. The set of reset times $\left\{t_{k}\right\}$ is an unbounded time sequence increasing monotonously with respect to $k, k \in \mathbb{Z}^{+}$, i.e., $t_{k}<t_{k+1}$ for any $k \in \mathbb{Z}^{+}$and $\lim _{k \rightarrow \infty} t_{k}=+\infty$. In conventional reset control, the set of reset times is defined as $\left\{t_{k}\right\} \triangleq$ $\left\{t_{k} \mid e\left(t_{k}\right)=0, t_{k}<t_{k+1}\right\}$, i.e., reset actions are triggered by the feedback error and additionally the states are always reset to zero. However, in our proposed reset controller (2), it is assumed that the reset times are prespecified and the reset values are modulated in terms of the system states and reference input for improved performance. Furthermore, we assume that the reset actions are finite in any finite time interval to ensure the existence of solutions.

Combining (1) and (2) gives the closed-loop system as follows:

$$
\begin{cases}\dot{x}=A x+B w, & t \neq t_{k} \\ x\left(t_{k}^{+}\right)=M_{k} x+N_{k} r, & t=t_{k} \\ y=C x+d & \end{cases}
$$

where $x=\left(x_{p}^{T}, x_{r}^{T}\right)^{T}, w=r-d-n$, and

$$
\begin{gathered}
A=\left[\begin{array}{cc}
A_{p}-B_{p} D_{r} C_{p} & B_{p} C_{r} \\
-B_{r} C_{p} & A_{r}
\end{array}\right], B=\left[\begin{array}{c}
B_{p} D_{r} \\
B_{r}
\end{array}\right], \\
M_{k}=\left[\begin{array}{cc}
I_{n_{p}} & 0 \\
E_{k} & F_{k}
\end{array}\right], N_{k}=\left[\begin{array}{c}
0 \\
G_{k}
\end{array}\right], C=\left[\begin{array}{ll}
C_{p} & 0
\end{array}\right],
\end{gathered}
$$

where $I_{n_{p}}$ is the identity matrix of dimension $n_{p}$.

\section{B. Stability Analysis}

In this subsection, we derive the stability condition of the reset control system (3) under the assumption of $r \equiv n \equiv$ $d \equiv 0$ but subject to non-zero initial condition. Hence, the reset control system (3) can be rewritten as

$$
\begin{cases}\dot{x}=A x, & t \neq t_{k}, \quad x(0)=x_{0} \\ x\left(t_{k}^{+}\right)=M_{k} x, & t=t_{k} .\end{cases}
$$

Suppose that the solution to (4) is continuous from the left, we thus have $x\left(t_{k}^{+}\right)=\lim _{t \rightarrow 0^{+}} x\left(t_{k}\right)=M_{k} x\left(t_{k}\right)$ and

$$
x\left(t_{k+1}^{+}\right)=M_{k+1} e^{A\left(t_{k+1}-t_{k}\right)} x\left(t_{k}^{+}\right) .
$$

Define $\eta_{k}=x\left(t_{k}^{+}\right), \Delta t_{k}=t_{k}-t_{k-1}, L_{k}=M_{k} e^{A \Delta t_{k}}$, and $t_{0}=0$, we have

$$
\eta_{k+1}=L_{k+1} \eta_{k}, \quad k=0,1, \cdots, N .
$$

The system (5) is referred to as the induced difference system of the impulsive system (4).

In general, in order to analyze the stability of system (4), we find a Lyapunov function of the base linear system $\dot{x}=A x$ which decreases at every jump time instant, i.e., find a positive function $V(x)$ such that $\dot{V}(x)=\left(\frac{\partial V(x)}{\partial x}\right)^{T} A x \leq$ $0, \Delta V(x)=V\left(M_{k} x\right)-V(x) \leq 0$. This indicates that the base linear system is stable and the impulses always decrease the amount of the Lyapunov function. The following result reveals that under some mild conditions, stability of an impulsive system is equivalent to stability of its induced difference system. Thus, the stability of the base linear system is not required any more, and the impulses are allowed to increase the value of Lyapunov function of the base linear system.

Proposition 1. If there exists a positive number $\Delta T>0$ such that $\Delta t_{k}=t_{k}-t_{k-1}<\Delta T$ for all $k \in \mathbb{Z}^{+}$, then the system (4) is (asymptotically) stable if and only if its induced difference system (5) is (asymptotically) stable.

Proof: The necessity is obvious. We only prove the sufficiency. According to the fact that the solutions to the base linear system $\dot{x}=A x$ depend continuously upon initial conditions, we have for any $\varepsilon>0$, there exists a positive number $\delta_{1}>0$ such that

$$
\left\|x_{0}\right\|<\delta_{1} \Rightarrow\left\|e^{A s} x_{0}\right\|<\varepsilon, \quad s \in[0, \Delta T] .
$$

Assume the induced difference system (5) is stable, then for $\delta_{1}$ selected above, there exists a positive number $\delta>0$ such that

$$
\left\|\eta_{0}\right\|<\delta \quad \Rightarrow \quad\left\|\eta_{k}\right\|<\delta_{1}, \quad k \in \mathbb{Z}^{+}
$$


Note that $\eta_{0}=x_{0} \triangleq x\left(0^{+}\right)$and for any $t \in[0,+\infty)$, there is a nonnegative integer $k$ such that $t \in\left(t_{k}, t_{k+1}\right]$, so

$$
x(t)=e^{A\left(t-t_{k}\right)} x\left(t_{k}^{+}\right)=e^{A\left(t-t_{k}\right)} \eta_{k} .
$$

Since $t-t_{k} \in[0, \Delta T]$, we have

$$
\|x(t)\|=\left\|e^{A\left(t-t_{k}\right)} \eta_{k}\right\|<\varepsilon, \quad t \in[0,+\infty) .
$$

So the system (4) is stable.

If the induced difference system is asymptotically stable, then we have

$$
\lim _{k \rightarrow \infty} \eta_{k}=0
$$

For any $\varepsilon>0$, choose $\delta_{1}$ such that

$$
\left\|x_{0}\right\|<\delta_{1} \Rightarrow\left\|e^{A s} x_{0}\right\|<\varepsilon, \quad s \in[0, \Delta T] .
$$

Thus for any $\eta_{0}\left(=x_{0}\right)$, there exists a $K\left(x_{0}\right) \in \mathbb{Z}^{+}$for

$$
\left\|\eta_{k}\right\|<\delta_{1}, \quad k \geq K\left(x_{0}\right)
$$

Then for any $t>K\left(x_{0}\right)$, there exists a $k \geq K\left(x_{0}\right)$ such that $t \in\left(t_{k}, t_{k+1}\right]$ and $x(t)=e^{A\left(t-t_{k}\right)} \eta_{k}$, so we have

$$
\|x(t)\|=\left\|e^{A\left(t-t_{k}\right)} \eta_{k}\right\|<\varepsilon
$$

due to $t-t_{k} \in[0, \Delta T]$. Therefore, $\lim _{t \rightarrow \infty} x(t)=0$, which implies that the system (4) is asymptotically stable.

Corollary 1. If both $\Delta t_{k}=\delta$ is a constant and $M_{k} \equiv M$ is a constant matrix, then the reset control system (4) is (asymptotically) stable if and only if

$$
\left|\lambda\left(M e^{A \delta}\right)\right| \leq 1, \quad(<1),
$$

where $\lambda(\cdot)$ denotes the eigenvalues of $(\cdot)$.

In practice, the base linear system is typically designed to be stable, thus the bounded constraint on $\left\{\Delta t_{k}\right\}$ can be relaxed. Thus we have the following.

Proposition 2. Assume the base linear system is stable, then the system (4) is (asymptotically) stable if and only if its induced difference system (5) is (asymptotically) stable. Proof : To complete the proof, just replace $\Delta T$ in the proof of Proposition 1 by $+\infty$ and follow the same lines.

\section{Reset Law Design for Improved Performance}

Consider the reset control system (3) and assume that the the base linear system has been appropriately designed for basic stability and performance, and the reset times are predefined. Our objective here is to find a set of reset values $x_{r}\left(t_{k}^{+}\right)$of the controller states such that the system transient response is improved. Suppose $n \equiv d \equiv 0$, we can formulate this problem as solving $x_{r}\left(t_{k}^{+}\right)$to minimize the following quadratic performance index

$$
\begin{gathered}
J_{k}=e^{T}\left(t_{k+1}\right) P_{0} e\left(t_{k+1}\right)+\dot{e}^{T}\left(t_{k+1}\right) Q_{0} \dot{e}\left(t_{k+1}\right) \\
+\int_{t_{k}}^{t_{k}+1} e(s)^{T} P_{1} e(s) d s
\end{gathered}
$$

where $e=r-y$, and $P_{0}, P_{1}$ and $Q_{0}$ are positive semidefinite matrices. To solve the problem, we assume that for any $r(t) \in \mathbb{R}$, there exists $x_{s s}=\left(x_{p s s}^{T}, x_{r s s}^{T}\right)^{T}$ such that

$$
\left\{\begin{aligned}
A x_{s s}+B r & =0 \\
C x_{s s}-r & =0
\end{aligned}\right.
$$

Define $\xi_{p}=x_{p}-x_{p s s}, \xi_{r}=x_{r}-x_{r s s}$, we have

$$
\begin{cases}\dot{\xi}=A \xi-\dot{x}_{s s}, & t \neq t_{k} \\ \xi_{r}\left(t_{k}^{+}\right)=\rho_{k}(\xi, r), & t=t_{k}\end{cases}
$$

where $\xi=\left(\xi_{p}^{T}, \xi_{r}^{T}\right)^{T}$. Hence we have $e=-C \xi, \dot{e}=-C A \xi$, and the performance index $J_{k}$ can thus be rewritten as

$$
J_{k}=\xi^{T}\left(t_{k+1}\right) \bar{P} \xi\left(t_{k+1}\right)+\int_{t_{k}}^{t_{k+1}} \xi^{T}(s) \bar{Q} \xi(s) d s
$$

where

$$
\begin{aligned}
\bar{P} & =C^{T} P_{0} C+A^{T} C^{T} Q_{0} C A \\
\bar{Q} & =C^{T} P_{1} C .
\end{aligned}
$$

If $\xi_{p}\left(t_{k}\right), r$ and $t_{k}, t_{k+1}$ are fixed, $J_{k}$ is in fact a function of $\xi_{r}\left(t_{k}^{+}\right)$. In order to choose $\xi_{r}\left(t_{k}^{+}\right)$such that $J_{k}$ is minimized, we need to calculate $\frac{\partial J_{k}}{\partial \xi_{r}\left(t_{k}^{+}\right)}$. In the following, we consider the case of $r$ being constant, which implies $\dot{x}_{s s}=0$, thus it gives that

$$
\xi(t)=e^{A t} \xi\left(t_{k}^{+}\right), \quad t \in\left(t_{k}, t_{k+1}\right] .
$$

We then have

$$
\begin{aligned}
& \frac{\partial J_{k}}{\partial \xi\left(t_{k}^{+}\right)} \\
= & \frac{\partial \xi\left(t_{k+1}\right)}{\partial \xi\left(t_{k}^{+}\right)} \frac{\partial}{\partial \xi\left(t_{k+1}\right)}\left(\xi^{T}\left(t_{k+1}\right) \bar{P} \xi\left(t_{k+1}\right)\right) \\
& +\int_{t_{k}}^{t_{k+1}} \frac{\partial \xi(s)}{\partial \xi\left(t_{k}^{+}\right)} \frac{\partial}{\partial \xi(s)}\left(\xi^{T}(s) \bar{Q} \xi(s)\right) d s \\
= & 2 e^{A^{T} \Delta t_{k}} \bar{P} e^{A \Delta t_{k}} \xi\left(t_{k}^{+}\right) \\
& +2 \int_{t_{k}}^{t_{k+1}} e^{A^{T}\left(s-t_{k}\right)} \bar{Q} e^{A\left(s-t_{k}\right)} \xi\left(t_{k}^{+}\right) d s \\
= & 2\left(e^{A^{T} \Delta t_{k}} \bar{P} e^{A \Delta t_{k}}\right. \\
& \left.+\int_{t_{k}}^{t_{k+1}} e^{A^{T}\left(s-t_{k}\right)} \bar{Q} e^{A\left(s-t_{k}\right)} d s\right) \xi\left(t_{k}^{+}\right) \\
= & 2\left(e^{A^{T} \Delta t_{k}} \bar{P} e^{A \Delta t_{k}}+\int_{0}^{\Delta t_{k}} e^{A^{T} s} \bar{Q} e^{A s} d s\right) \xi\left(t_{k}^{+}\right) \\
= & 2 \Gamma_{k} \xi\left(t_{k}^{+}\right)
\end{aligned}
$$

where

$$
\Gamma_{k}=e^{A^{T} \Delta t_{k}} \bar{P} e^{A \Delta t_{k}}+\int_{0}^{\Delta t_{k}} e^{A^{T} s} \bar{Q} e^{A s} d s .
$$

Partition $\Gamma_{k}$ as

$$
\Gamma_{k}=\left(\begin{array}{cc}
\Gamma_{k}^{11} & \Gamma_{k}^{12} \\
\Gamma_{k}^{21} & \Gamma_{k}^{22}
\end{array}\right)
$$

with $\Gamma_{k}^{12}=\left(\Gamma_{k}^{21}\right)^{T}$. Thus we have

$$
\begin{aligned}
\frac{\partial J_{k}}{\partial \xi_{r}\left(t_{k}^{+}\right)} & =\frac{\partial \xi\left(t_{k}^{+}\right)}{\partial \xi_{r}\left(t_{k}^{+}\right)} \frac{\partial J_{k}}{\partial \xi\left(t_{k}^{+}\right)} \\
& =2\left(\begin{array}{cc}
0 & I
\end{array}\right)\left(\begin{array}{cc}
\Gamma_{k}^{11} & \Gamma_{k}^{12} \\
\Gamma_{k}^{21} & \Gamma_{k}^{22}
\end{array}\right) \xi\left(t_{k}^{+}\right) \\
& =2\left(\Gamma_{k}^{21} \xi_{p}\left(t_{k}\right)+\Gamma_{k}^{22} \xi_{r}\left(t_{k}^{+}\right)\right)
\end{aligned}
$$


If $\Gamma_{k}^{22}$ is positive definite, letting $\frac{\partial J_{k}}{\partial \xi_{r}\left(t_{k}^{+}\right)}=0$ derives

$$
\xi_{r}\left(t_{k}^{+}\right)=-\left(\Gamma_{k}^{22}\right)^{-1} \Gamma_{k}^{21} \xi_{p}\left(t_{k}\right) .
$$

Thus the reset law which minimizes $J_{k}$ is given by

$$
x_{r}\left(t_{k}^{+}\right)=-\left(\Gamma_{k}^{22}\right)^{-1} \Gamma_{k}^{21}\left(x_{p}-x_{p s s}\right)+x_{r s s} .
$$

Furthermore, if we consider equidistant reset control (i.e., $\Delta t_{k} \equiv \delta$ is a constant), then $\Gamma_{k} \equiv \Gamma$ is a constant matrix independent on $k$. According to the analysis above and by Corollary 1 , we have the following.

Proposition 3. Suppose that $\Delta t_{k}=\delta$ is a constant and $\Gamma^{22}>0$, then the reset law which minimizes $J_{k}(8)$ is independent on $k$ and is given by

$$
x_{r}\left(t_{k}^{+}\right)=-\left(\Gamma^{22}\right)^{-1} \Gamma^{21}\left(x_{p}-x_{p s s}\right)+x_{r s s} .
$$

In addition, the corresponding closed-loop system (4) under this reset law is asymptotically stable if and only if

$$
\left|\lambda\left(\left(\begin{array}{cc}
I_{n_{p}} & 0 \\
-\left(\Gamma^{22}\right)^{-1} \Gamma^{21} & 0
\end{array}\right) e^{A \delta}\right)\right|<1 .
$$

Remark : If the stability condition (20) can not be satisfied, we have to retune the base controller or the reset law design parameters. Generally, the base linear system is designed to be asymptotically stable, then we can alternatively use the following reset law

$$
\begin{aligned}
x_{r}\left(t_{k}^{+}\right)= & -\mu\left[\left(\Gamma^{22}\right)^{-1} \Gamma^{21}\left(x_{p}-x_{p s s}\right)+x_{r s s}\right] \\
& +(1-\mu) x_{r}, \quad \mu \in[0,1],
\end{aligned}
$$

to compromise between the stability and the performance characterized by $J_{k}$. It is clear that when $\mu$ varies from 1 to 0 , the reset control system tends to the base linear system that is assumed asymptotically stable. On the other hand, according to (16), we have

$$
\begin{aligned}
\frac{\partial J_{k}}{\partial \mu}= & \frac{\partial \xi_{r}\left(t_{k}^{+}\right)}{\partial \mu} \frac{\partial J_{k}}{\partial \xi_{r}\left(t_{k}^{+}\right)} \\
= & -2(1-\mu)\left[\left(\Gamma^{22}\right)^{-1} \Gamma^{21} \xi_{p}\left(t_{k}\right)+\xi_{r}\left(t_{k}\right)\right]^{T} \\
& \times \Gamma^{22}\left[\left(\Gamma^{22}\right)^{-1} \Gamma^{21} \xi_{p}\left(t_{k}\right)+\xi_{r}\left(t_{k}\right)\right] \\
\leq & 0 .
\end{aligned}
$$

Denote $x_{r}\left(t_{k}^{+}\right)=\rho(\xi, r, \mu)$, thus $J_{k}(\rho(\xi, r, \mu))$ is monotonously decreasing when $\mu$ varies from 0 to 1 . Thus we can always choose $\mu \in(0,1)$ such that the closed-loop system is asymptotically stable and at the same time,

$$
J_{k}(\rho(\xi, r, 1))<J_{k}(\rho(\xi, r, \mu))<J_{k}(\rho(\xi, r, 0)) .
$$

The above inequality indicates that the performance index of the resulting reset control system is always less than that of the base linear system, though the minimal index can not be achieved.

\section{EXPERIMENTAL RESULTS}

In this section, we experimentally verify the proposed improved reset control design on a PZT microactuator positioning stage (P-752 PZT Flexure Stage System, Polytec PI, Germany) as shown in Fig. 2.

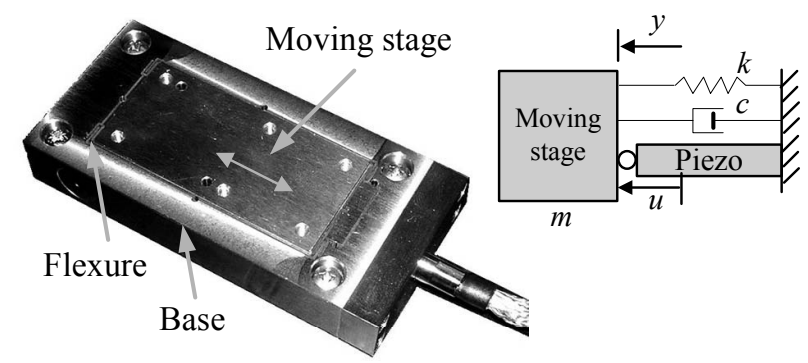

Fig. 2. A picture of the PZT microactuator positioning stage.

\section{A. Modeling of the PZT Positioning Stage}

The PZT positioning stage consists of a PZT microactuator, a moving stage connected with the base via the flexures, a PZT amplifier, and an integrated capacitive position feedback sensor with $0.2 \mathrm{~nm}$ resolution to measure the displacement of the moving stage. The PZT microactuator is of high stiffness and has a maximum travel range of $\pm 15 \mu \mathrm{m}$. The mechanical resonance caused by the flexures is actively damped by the integrated control electronics. Thus, the dynamics of the PZT positioning stage can be simply depicted by a massdamper-spring system as shown in Fig. 2, which can be then described by a state space form as follows:

$$
\Sigma_{P}:\left\{\begin{aligned}
\dot{x}_{1} & =x_{2} \\
\dot{x}_{2} & =-a_{1} x_{1}-a_{2} x_{2}+b u \\
y & =x_{1}
\end{aligned}\right.
$$

where $x_{1}, x_{2}$ are the position and velocity of the moving stage, respectively, and $u$ is the control input to the PZT amplifier. The modal parameters in (22) are identified from experimental frequency response data. A dynamic signal analyser (HP 35670A, Hewlett Packard Company, Washington) is used to generate the swept-sinusoidal excitation signals and collect the frequency response data from the excitation signals $u$ to the position output $y$. The dashed lines in Fig. 3 show the measured frequency responses of the PZT positioning stage. The PZT dynamics is of high stiffness that exhibits a flat gain in the low frequency range. By using the least square estimation method [17], we obtain the model parameters as follows:

$$
a_{1}=10^{6}, a_{2}=1810, b=3 \times 10^{6} .
$$

The solid lines in Fig. 3 show that the identified model match the measured model well in the frequency range of interest.

\section{B. Reset Control Law}

Our objective is to design a control law for fast step responses with little overshoot and no steady-state error, for which high open-loop gains in low frequency range and high bandwidth with sufficient stability margin are typically required. Thus, we use the classical proportional-integral (PI) controller as the base controller,

$$
\frac{u(s)}{e(s)}=k_{p}+\frac{k_{i}}{s},
$$

where $k_{p}=0.08$ and $k_{i}=300$. The base linear system has an open-loop bandwidth $109 \mathrm{~Hz}$, and gain/phase margin 

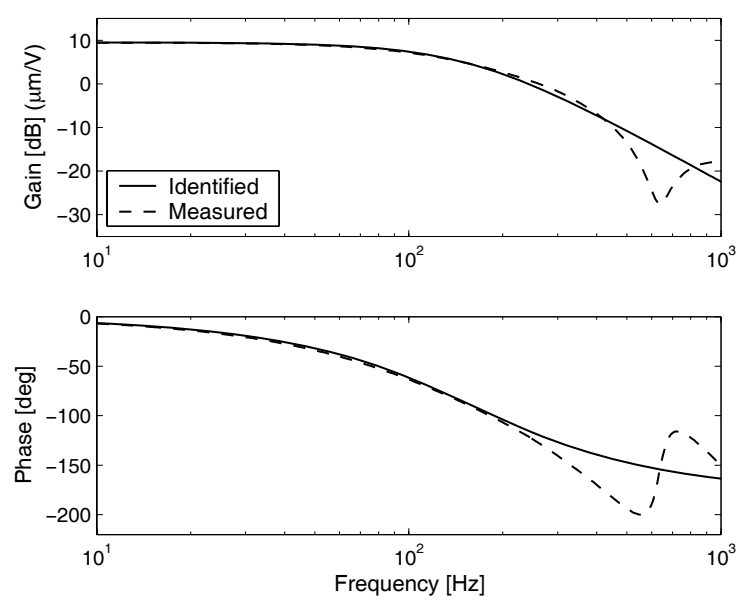

Fig. 3. Frequency responses of the PZT microactuator positioning stage.

$11 \mathrm{~dB} / 33$ deg. The integrator increases the low frequency gains to achieve a fast rise time and remove the steady-state error (see the dashed lines in Fig. 4). However, the overshoot induced by the integrator is also significant $(40 \%$ of the step level), which results in tedious settling time. Therefore, it is expected that the overshoot could be reduced by resetting the integrator state to the proper values calculated by using Proposition 3.

Here, we set the reset time interval as a constant, i,e., $\Delta t_{k}=1 \mathrm{~ms}$ and select the tuning parameters of $J_{k}$ in (8) as $P_{0}=2.1, Q_{0}=1.0 \times 10^{-6}, P_{1}=0$. Thus, we can easily obtain $x_{s s}$ and $\Gamma$ by calculating (9) and (15), respectively. The resulting reset controller is then described in state space as follows:

$$
\begin{cases}\dot{x}_{r}=e, & t \neq t_{k} \\ x_{r}\left(t_{k}^{+}\right)=E_{1} x_{1}+E_{2} x_{2}+G r, & t=t_{k}, \\ u=k_{i} x_{r}+k_{p} e & \end{cases}
$$

where $E_{1}=-2.8 \times 10^{-4}, E_{2}=-6.8 \times 10^{-7}, G=0.0014$, $k_{i}=300$, and $k_{p}=0.08$. Moreover, it is easy to verify that the resulting reset control system satisfies the stability condition in (20), which implies that the closed-loop system is asymptotically stable.

\section{Results and Discussion}

The reset controller (24) was implemented by a real-time DSP system (dSPACE-DS1103) with the sampling time of $T_{s}=50 \mu \mathrm{s}$. The velocity $x_{2}$ is estimated by the backward differentiation of the measurable position signal $x_{1}(t)$, i.e., $x_{2}(z)=\frac{z-1}{T_{s} z} x_{1}(z)$.

The experimental results for $1 \mu \mathrm{m}$ step responses are shown in Fig. 4. It can be seen that our improved reset control completely removes the overshoot and thus reduces the settling time from $15 \mathrm{~ms}$ (No reset) to $3 \mathrm{~ms}$. Moreover, we observe that the improved reset control has a faster transient response compared to that under no reset because the integrator state was reset to minimize $J_{k}$ from the beginning at $t=0$ leading to a larger control input at the initial stage and thus faster response. When the position output approaches the target, the integrator state is reset
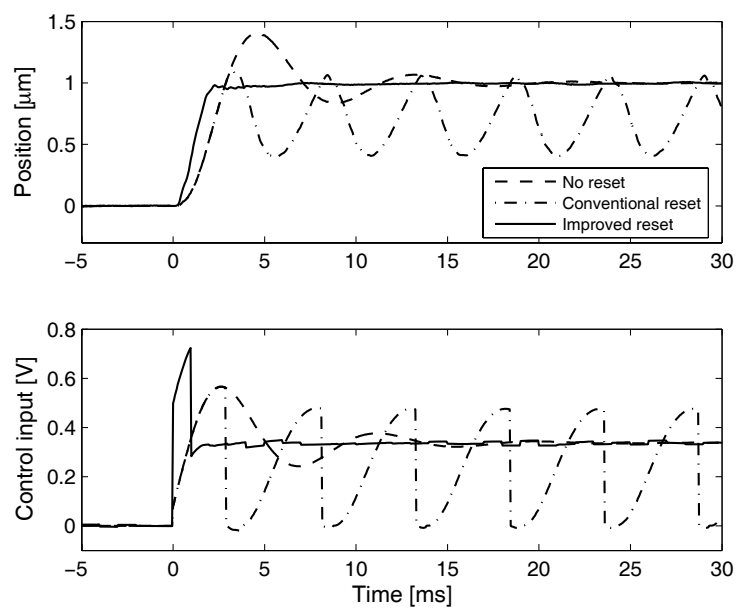

Fig. 4. Step responses $(r=1 \mu \mathrm{m})$ of the reset control system. The improved reset control exhibits little overshoot and the resultant settling time is $3 \mathrm{~ms}$, which is identical to the rise time of the base linear system (No reset). The conventional reset control still exhibits $15 \%$ overshoot in the first peak and causes limit cycles in steady state.
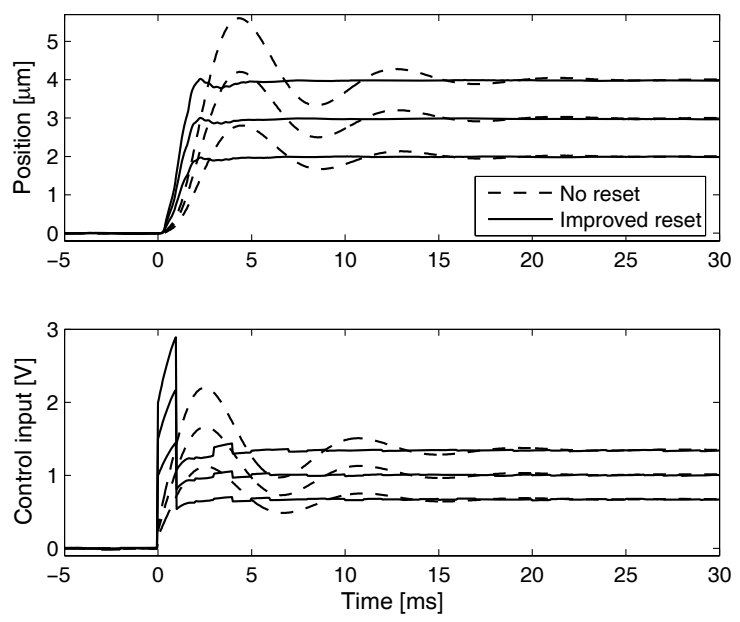

Fig. 5. Time responses of the reset control system to various step levels $(r=2,3,4 \mu \mathrm{m})$. The overshoots in all cases are nearly removed and the settling times are maintained to be $3 \mathrm{~ms}$.

to a smaller value (see the control input at $t=1 \mathrm{~ms}$ in Fig. 4) to reduce the overshoot and keep the moving stage at the desired position. In this case, the conventional reset control works badly, which can only partially reduces the first overshoot peak and results in limit cycles. This is because resetting the integrator state to zero tends to resetting the control input to zero, which will cause the moving stage going to its initial position due to the high stiffness of the PZT actuator. Thus, the conventional reset control needs an intentional interplay between the reset mechanism and an appropriately designed base linear controller [7].

Next, we test the robustness of the improved reset control system against various step levels, disturbance and sensor noise. Fig. 5 shows the time responses to various step levels. Figs. 6 and 7 respectively show the time responses to a single-frequency $(100 \mathrm{~Hz})$ sinusoidal input disturbance $u_{d}$ and sensor noise $n$, which are artifically introduced to 

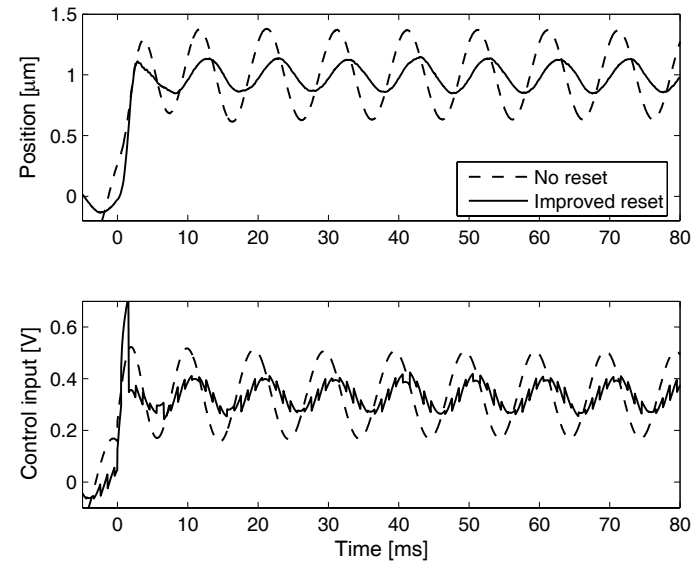

Fig. 6. Time responses of the reset control system to step input $r=1 \mu \mathrm{m}$ and sinusoidal input disturbance $u_{d}=0.1 \sin (2 \pi 100 t) \mathrm{V}$.
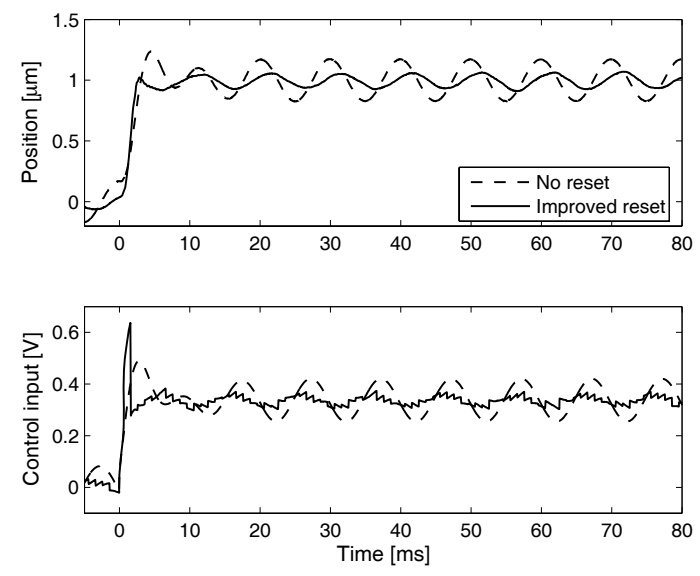

Fig. 7. Time responses of the reset control system to step input $r=1 \mu \mathrm{m}$ and sinusoidal sensor noise $n=0.1 \sin (2 \pi 100 t) \mu \mathrm{m}$.

the control system. We can see that the improved reset control simultaneously provides an improvement of $65 \%$ in both disturbance and noise suppression based on the base linear system (No reset). However, we also observe that this favorable property does not hold in other frequencies or there even exists performance degradation as shown in Fig. 8.

\section{CONCLUSIONS AND FUTURE WORKS}

This paper studied an improved reset control system, where the reset times are prespecified and the controller states are reset to certain non-zero values. The stability condition of the reset control system is given and the reset law design is presented. Experimental results on a PZT microactuator positioning stage showed that the improved reset control can achieve faster settling time than the conventional reset control by removing the overshoot. Moreover, it can simultaneously achieve sinusoidal disturbance and sensor noise suppression at some frequencies. Our future works will include the design of the reset time instants and disturbance and noise suppression in a wide frequency range to enhance the overall system performance.
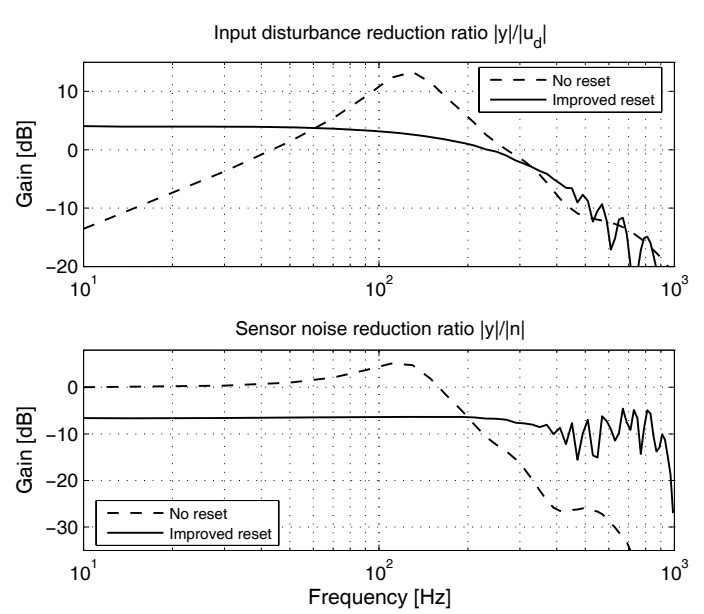

Fig. 8. Plot of reduction ratio of sinusoidal input disturbance and sensor noise versus frequency. The improved reset control adversely increases the low-frequency disturbance and high-frequency noise reduction ratio. This problem will be investigated and improved in our future works.

\section{REFERENCES}

[1] J. Clegg, "A nonlinear integrator for servomechanism," Trans. AIEE, vol. 77, pp. 41-42, 1958.

[2] K. Krishman and I. Horowitz, "Synthesis of a nonlinear feedback system with significant plant-ignorance for prescribed system tolerance," Int. J. Control, vol. 19, pp. 689-706, 1974.

[3] I. Horowitz and P. Rosenbaum, "Nonlinear design for cost of feedback reduction in systems with large parameter uncertainty," Int. J. Control, vol. 21, pp. 977-1001, 1975.

[4] Y. Chait and C. Hollot, "On Horowitz's contributions to reset control," Int. J. Robust Nonlinear Control, vol. 12, pp. 335-355, 2002.

[5] L. Zaccarian, D. Nesic, and A. Teel, "First order reset elements and the Clegg integrator revisited," in Proc. of American Control Conference, 2005, pp. 563-568.

[6] O. Beker, C. Hollot, and Y. Chait, "Plant with integrator: an example of reset control overcoming limitations of linear feedback," IEEE Trans. Automatic Control, vol. 46, no. 11, pp. 1797-1799, 2001.

[7] Y. Zheng, Y. Chait, C. Hollot, M. Steinbuch, and M. Norg, "Experimental demonstration of reset control design," Control Engineering Practice, vol. 8, pp. 113-120, 2000.

[8] D. Wu, G. Guo, and Y. Wang, "Reset integral-derivative control for HDD servo systems," IEEE Trans. Contr. Syst. Technol., vol. 15, no. 1, pp. 161-167, Jan. 2007.

[9] J. Bobrow, F. Jabbari, and K. Thai, "An active truss element and control law for vibration suppression," Smart Materials and Structures, vol. 4, pp. 264-269, 1995.

[10] R. Bupp, D. Bernstein, V. Chellaboina, and W. Haddad, "Resetting virtual absorbers for vibration control," in Proc. of American Control Conference, 1997, pp. 2647-2651.

[11] Q. Chen, Y. Chait, and C. Hollot, "Analysis of reset control systems consisting of a FORE and second order loop," Journal of Dynamic systems, Measurement and Control, vol. 123, pp. 279-283, 2001.

[12] O. Beker, C. Hollot, Q. Chen, and Y. Chait, "Stability of a reset control system under constant inputs," in Proc. of American Control Conference, 1999, pp. 3044-3045.

[13] O. Beker, C. Hollot, and Y. Chait, "Stability of a MIMO reset control system under constant inputs," in Proc. IEEE Conf. Decision and Control, 1999, pp. 2780-2781.

[14] Q. Chen, C. Hollot, Y. Chait, "Stability and asymptotic performance analysis of a class reset control systems," in Proc. IEEE Conf. Decision and Control, 2000, pp. 251-256.

[15] O. Beker, C. Hollot, Y. Chait, and H. Han, "Fundamental properties of reset control systems." Automatica, vol. 40, pp. 905-915, 2004.

[16] T. Yang, Impulsive Control Theory, Lecture notes on control and information sciences, Springer-Verlag Berlin Hedelberg NY, vol. 272, 2001.

[17] L. Ljung, System Identification - Theory For the User, 2rd ed., PTR Prentice Hall, 1999. 